\title{
The dynamic compressive behavior of beryllium bearing bulk metallic glasses
}

\author{
H. A. Bruck \\ Idaho National Engineering Labs, Mail Stop 2218, Idaho Falls, Idaho 83415-2218
}

\author{
A. J. Rosakis \\ Department of Aeronautics, California Institute of Technology, Mail Stop 105-50, \\ Pasadena, California 91125 \\ W. L. Johnson \\ Department of Materials Science, California Institute of Technology, Mail Stop 105-50, \\ Pasadena, California 91125
}

(Received 14 March 1995; accepted 26 October 1995)

\begin{abstract}
In 1993, a new beryllium bearing bulk metallic glass with the nominal composition of $\mathrm{Zr}_{41.25} \mathrm{Ti}_{13.75} \mathrm{Cu}_{12.5} \mathrm{Ni}_{10} \mathrm{Be}_{22.5}$ was discovered at Caltech. This metallic glass can be cast as cylindrical rods as large as $16 \mathrm{~mm}$ in diameter, which permitted specimens to be fabricated with geometries suitable for dynamic testing. For the first time, the dynamic compressive yield behavior of a metallic glass was characterized at strain rates of $10^{2}$ to $10^{4} / \mathrm{s}$ by using the split Hopkinson pressure bar. A high-speed infrared thermal detector was also used to determine if adiabatic heating occurred during dynamic deformation of the metallic glass. From these tests it appears that the yield stress of the metallic glass is insensitive to strain rate and no adiabatic heating occurs before yielding.
\end{abstract}

\section{INTRODUCTION}

Many components formed from high strength alloys experience dynamic mechanical loading during their fabrication and use, which cause the materials to deform at very high strain rates. In order to understand the limitations of using high strength alloys in these processes, it is necessary to quantify the material's dynamic constitutive behavior. Insight into this behavior has been customarily obtained using Taylor anvil tests ${ }^{1}$ or Kolsky bars. ${ }^{2}$

One class of high strength alloys that can be potentially used in many commercial and industrial applications is known as amorphous metal alloys or metallic glasses. Previously, cooling rate requirements have limited the size of metallic glass specimens available for mechanical testing to cylindrical rods no larger than $2 \mathrm{~mm}$ in diameter. Larger specimens are required to obtain reliable data on the mechanical behavior of these alloys. Recently, metallic glasses containing beryllium have been fabricated as ingots up to $16 \mathrm{~mm}$ in diameter by directly quenching from the melt. ${ }^{3}$ Mechanical tests have been conducted on specimens manufactured from these beryllium bearing bulk metallic glasses. ${ }^{4}$ The quasistatic constitutive behavior of these bulk metallic glasses was characterized in these tests.

At that time, all of the constitutive characterization of metallic glasses was performed in quasistatic tests. No data are currently available on the dynamic constitutive behavior of metallic glasses. An attempt was made by Cline and Reaugh ${ }^{5}$ to determine the dynamic yield strength of a palladium alloy metallic glass using the Taylor anvil test; however, they concluded that this was not appropriate.

The goal of this work is to characterize the dynamic compressive yield behavior of the $\mathrm{Zr}_{41.25} \mathrm{Ti}_{13.75} \mathrm{Cu}_{12.5}$ $\mathrm{Ni}_{10} \mathrm{Be}_{22.5}$ metallic glass using a Kolsky bar. Thermal measurements were also made using a high speed infrared temperature sensor ${ }^{6}$ to investigate whether adiabatic heating occurs during the dynamic deformation of the glass.

\section{KOLSKY BAR THEORY}

The basic principle of the Kolsky bar is illustrated in Fig. 1(a). The setup basically consists of two elastic pressure bars, $\overline{A B}$ known as the incident or input bar and $\overline{C D}$ known as the transmitter or output bar. A specimen is placed between the two bars. A striker bar impacts the input bar at $\bar{A}$. This sets up a pressure pulse within the incident bar with a strain $\epsilon_{I}(t)$. When this pulse reaches $\bar{B}$, the end of the incident bar, part of the wave is reflected with a strain $\epsilon_{R}(t)$ and part of the wave is transmitted to the output bar with a strain $\epsilon_{T}(t)$. Strain gauges are placed in the middle of the elastic pressure bars to provide the time-resolved signals of the incident, reflected, and transmitted pulses. The pulse is reflected from the interface of the bar and specimen at $\bar{B}$ due to impedance mismatch.

When the specimen is deforming uniformly, the strain rate $\dot{\epsilon}$ within the specimen is proportional to the 


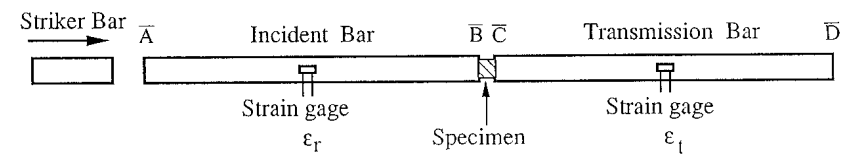

(a)

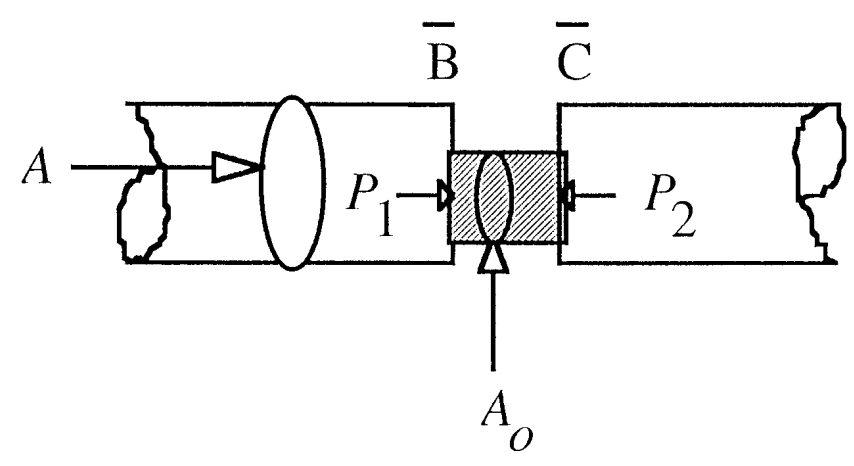

(b)

FIG. 1. (a) Kolsky bar used for dynamic compression tests. (b) Stress and strain analysis of homogeneously deformed specimen.

reflected wave's amplitude. Similarly, the stress within the sample is proportional to the amplitude of the transmitted wave. This can be shown by the following analysis [Fig. 1(b)]. In this figure, $\bar{A}$ is the cross-sectional area of the elastic bars and $A_{0}$ is the cross-sectional area of the specimen. The strain rate in the deforming specimen is

$$
\frac{d \epsilon}{d t}=\dot{\epsilon}(t)=\frac{v_{1}(t)-v_{2}(t)}{L},
$$

where $v_{1}$ and $v_{2}$ are particle velocities at $\bar{B}$ and $\bar{C}$, respectively, and $L$ is the length of the specimen. From characteristic relations for one-dimensional wave propagation, assuming no wave dispersion occurs, one has

$$
\begin{aligned}
& v_{1}=c_{0}\left(\epsilon_{I}-\epsilon_{R}\right), \\
& v_{2}=c_{0} \epsilon_{T},
\end{aligned}
$$

where $c_{0}=\sqrt{E / \rho}$ is the longitudinal bar wave speed for the elastic bars, $E$ is Young's modulus of the elastic bars, and $\rho$ is the density of the elastic bars. From Eqs. (1) and (2):

$$
\dot{\epsilon}(t)=\frac{c_{0}}{L}\left[\epsilon_{1}(t)-\epsilon_{R}(t)-\epsilon_{T}(t)\right] .
$$

The average stress in the specimen is

$$
\sigma(t)=\frac{P_{1}(t)+P_{2}(t)}{2 A_{0}},
$$

where $P_{1}$ and $P_{2}$ are the forces at the incident bar/specimen and specimen/transmitter bar interfaces, respectively. Assuming that no wave dispersion occurs:

$$
\begin{aligned}
& P_{1}=E A_{0}\left[\epsilon_{I}(t)+\epsilon_{R}(t)\right], \\
& P_{2}=E A_{0} \epsilon_{T}(t) .
\end{aligned}
$$

From Eqs. (4) and (5):

$$
\sigma(t)=\frac{E A_{0}}{2 A}\left[\epsilon_{I}(t)+\epsilon_{R}(t)+\epsilon_{T}(t)\right] .
$$

When the specimen is deforming uniformly (homogeneously), the stress at the incident bar/specimen interface equals the stress at the transmitter bar/specimen interface. From Eq. (5):

$$
P_{1}(t)=P_{2}(t)
$$

which implies that:

$$
\epsilon_{I}(t)+\epsilon_{R}(t)=\epsilon_{T}(t) .
$$

From Eqs. (3), (6), and (8):

$$
\begin{aligned}
\sigma(t) & =\frac{E A_{0}}{A} \epsilon_{T}(t), \\
\dot{\boldsymbol{\epsilon}}(t) & =-\frac{2 c_{0}}{L} \epsilon_{R}(t) .
\end{aligned}
$$

Integrating Eq. (10), we obtain the time history of the strain as follows:

$$
\epsilon(t)=\int_{0}^{t} \dot{\epsilon}(\tau) d \tau .
$$

The stress-strain behavior is completely determined by measuring $\epsilon_{T}(t)$ and $\epsilon_{R}(t)$ on the elastic pressure bars using strain gauges.

\section{HIGH-SPEED INFRARED THERMAL MEASUREMENTS}

High-speed, noncontact techniques have been developed for measuring temperature fields on the surfaces of deforming objects. ${ }^{6,7}$ These techniques use detectors to measure the infrared thermal signatures generated by the deforming object. An Offner imaging system first employed by Zehnder and Rosakis ${ }^{6}$ was used for the dynamic compression tests to focus the surface of the compression specimens onto a single infrared detector. The Offner system has a fixed 1:1 magnification, so that optics were used with a focal length of approximately $160 \mathrm{~mm}$ and an object distance of $320 \mathrm{~mm}$ in order to protect the optics by keeping them a sufficient distance away from the test specimen.

A single EG\&G J15D12 HgCdTe infrared detector with dimensions $100 \mu \mathrm{m}$ square and a temperature range of approximately 300 to $1000 \mathrm{~K}$ was used to detect the temperature. The HgCdTe detector was cooled with liquid nitrogen, and the voltage from the detector was outputted to a preamp with a bandpass of $5 \mathrm{~Hz}$ to $1 \mathrm{MHz}$, well beyond the detector's cutoff frequency of $400 \mathrm{kHz}$. Because the sensor had a very high signal-to-noise ration, the preamp was connected to an amplifier set at $20 \times$ and a filter set at $10 \mathrm{kHz}$, which in turn was connected to a Nicolet 440 digital oscilloscope that was connected to the strain gauges on the Kolsky bars. 
The $\mathrm{HgCdTe}$ detector integrates wavelengths ranging from $2 \mu \mathrm{m}$ to $12 \mu \mathrm{m}$. The voltage produced by the detectors is related to the energy emitted by the specimen through an integral, depending upon the emissivity of the material, $\epsilon(\lambda, T)$, the spectral responsivity of the $\mathrm{HgCdTe}$ detector, $R(\lambda)$, and the black body radiation $P(\lambda)$, as follows ${ }^{7}$ :

$$
\begin{aligned}
v\left(T, T_{0}\right)= & A A_{D} \beta \\
& \times \int_{2 \mu \mathrm{m}}^{15 \mu \mathrm{m}} R(\lambda)[P(\lambda, T) \epsilon(\lambda, T) \\
& \left.\quad-P\left(\lambda, T_{0}\right) \epsilon\left(\lambda, T_{0}\right)\right] d \lambda,
\end{aligned}
$$

where the integration variable $\lambda$ is the wavelength of the radiation, $A$ is the amplification, $A_{D}$ is the detector area, and $\beta$ is the fraction of energy transmitted to the detectors by the optical system (related to the aperture). When evaluated for $\mathrm{HgCdTe}$ detectors, this relationship is nearly linear on a log-log plot of voltage versus temperatures. In order to determine this relationship for the $\mathrm{Zr}_{41.25} \mathrm{Ti}_{13.75} \mathrm{Cu}_{12.5} \mathrm{Ni}_{10} \mathrm{Be}_{22.5}$ metallic glass, a $1.5 \mathrm{~mm}$ thick plate specimen with a thermocouple clamped to its surface was heated in an oven. The voltage output from the detectors and the thermocouple readings were then recorded as the specimen cooled. This calibration datum was then plotted on a log-log plot, and the linear relationship was determined (Fig. 2). This relationship was then used to convert the voltage record from the detector into a temperature measurement.

\section{EXPERIMENTAL PROCEDURE}

Kolsky bar specimens were fabricated as cylindrical rods with the nominal composition $\mathrm{Zr}_{41.25} \mathrm{Ti}_{13.75} \mathrm{Cu}_{12.5^{-}}$ $\mathrm{Ni}_{10} \mathrm{Be}_{22.5}$ using commercially pure $(>99.5 \%)$ elements. The rods were cast by injecting the molten alloy into a copper mold with cylindrical cavities that are $2.5 \mathrm{~mm}$ in diameter and $30 \mathrm{~mm}$ long. This geometry was chosen because of the ease with which it could be fabricated

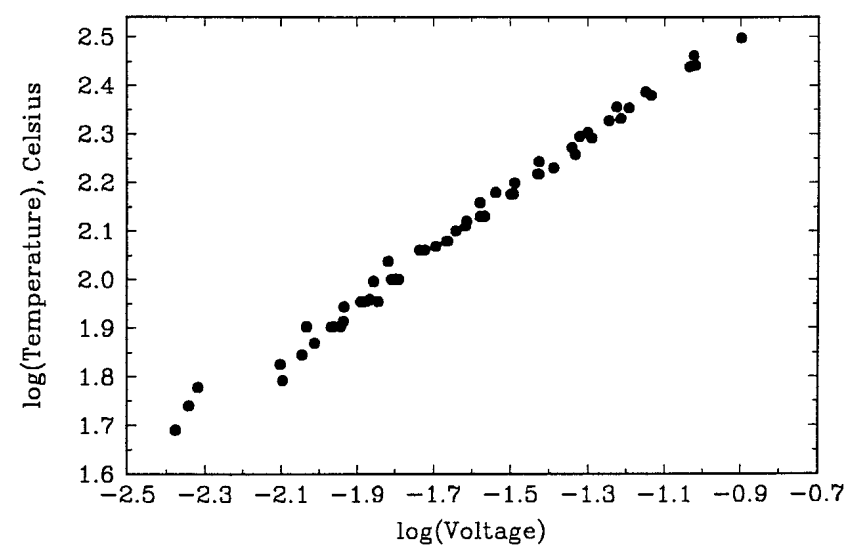

FIG. 2. Thermal calibration curve for $\mathrm{Zr}_{41.25} \mathrm{Ti}_{13.75} \mathrm{Cu}_{12.5} \mathrm{Ni}_{10} \mathrm{Be}_{22.5}$. and the significant ductility it exhibited in quasistatic compression tests. ${ }^{4}$ Details of the alloy design and casting procedure can be found in Ref. 3. Periodically, disks of approximately $250 \mu \mathrm{m}$ in thickness were machined from the rods and placed in an Inel CPS-120 $\mathrm{x}$-ray diffractometer to verify that the structure was amorphous.

After casting the rods, Kolsky bar specimens were machined with length to diameter $(l / d)$ aspect ratios ranging from $1: 2$ to $2: 1$. The ends of the specimens were then mechanically polished with 600 grit grinding paper using a V-block to ensure flat and parallel surfaces. By measuring the length of the specimen at four different locations along the specimen's edge with a Mitutoyo micrometer, the surfaces were found to be flat and parallel to within $3 \mu \mathrm{m}$, the resolution of the micrometer.

The dynamic testing of the $\mathrm{Zr}_{41.25} \mathrm{Ti}_{13.75} \mathrm{Cu}_{12.5} \mathrm{Ni}_{10^{-}}$ $\mathrm{Be}_{22.5}$ metallic glass was conducted using a split Hopkinson bar with elastic bars made from C350 maraging steel that has a yield stress of $2.5 \mathrm{GPa}$. The bars were 1.22 meters in length and $1.27 \mathrm{~cm}$ in diameter. Specimens were placed between the elastic bars using a pair of WC insets coated with "Molygraph Extreme Pressure Multi-purpose" grease to reduce end effects. The WC inserts were machined to a diameter of approximately $0.85 \mathrm{~cm}$ in order to impedance match them with the C350 maraging steel. A striker bar was used that was manufactured from the same material as the elastic bars and with the same diameter, but only $10 \mathrm{~cm}$ in length. Strain gauges from Micromeasurements Group were placed midway from the ends of the elastic bars to measure the strain response on the surface of the bar from the propagating waves. The strain gauges were attached to quarter Wheatstone bridges whose voltage outputs were measured with a Nicolet 440 digital oscilloscope using a sampling rate of $5 \mathrm{MHz}$.

Sometimes when the striker bar impacts the incident bar, there is some misalignment which can cause a variation in the amplitude of the incident stress pulse often referred to as "ringing." Consequently, there is some dispersion of the stress pulse that is detected by the strain gauges well after the stress pulse has passed underneath the strain gage. This makes it extremely difficult to detect the start of the reflected wave when it passes through the same strain gage.

In order to reduce dispersion from the incident stress pulse, a copper insert was placed between the striker bar and the incident bar as described by Follansbee in the Metals Handbook. ${ }^{8}$ The resulting stress pulse generated by using the copper inserts was a ramp function instead of the step function generated when the copper inserts are removed. The period of the stress pulse was nearly quadrupled by the presence of the copper inserts. Theoretically, the length of the stress pulse would be twice the length of the striker bar, which implies that 
the stress pulse generated using copper inserts would be eight times the length of the striker bar. A constraint on the length of the stress pulse is it should be less than the length of the bar if there is to be no overlap of incident and reflected signals underneath the strain gage when it is positioned midway from the ends of the bar. So, the striker bar should be less than $15 \mathrm{~cm}$ in length if the stress pulse is to be less than the length of the elastic bar, which dictated that the $10 \mathrm{~cm}$ long striker bar be used.

Striker bar velocities varied from $5 \mathrm{~m} / \mathrm{s}$ to $20 \mathrm{~m} / \mathrm{s}$, which generated strain rates in the specimen of 300 to $6000 / \mathrm{s}$, depending on the length of the specimen. One problem with using the copper inserts was the stress pulses generated by their deformations were of insufficient amplitude to obtain strain rates in the specimen greater than about $2000 / \mathrm{s}$ to $3000 / \mathrm{s}$, depending on the length of the specimen. In order to obtain the higher strain rates, it was necessary to remove the copper inserts.

\section{EXPERIMENTAL RESULTS AND DISCUSSION}

Strain gage data and the thermal measurements from a typical Kolsky bar test on the $\mathrm{Zr}_{41.25} \mathrm{Ti}_{13.75} \mathrm{Cu}_{12.5^{-}}$ $\mathrm{Ni}_{10} \mathrm{Be}_{22.5}$ metallic glass can be seen in Figs. 3 and 4. The dynamic stress-strain curve and strain-strain rate curve reduced from these signals can be seen in Figs. 5 and 6. Yield stresses were obtained for a variety of strain rates and plotted in Fig. 7. Catastrophic failure of dynamic compression specimens occurred due to shear banding along a plane oriented $45^{\circ}$ to the loading axis as seen in Fig. 8, which is identical to the failure observed in quasistatic tests.

Thermal measurements indicated that temperatures varied dramatically after the specimen failed, reaching peak values of approximately $500{ }^{\circ} \mathrm{C}$. Since the specimen failed by shear binding, it is possible that the variations in the thermal trace are due to the shear band passing through the detector and that the peak values correspond to the maximum temperature reached within

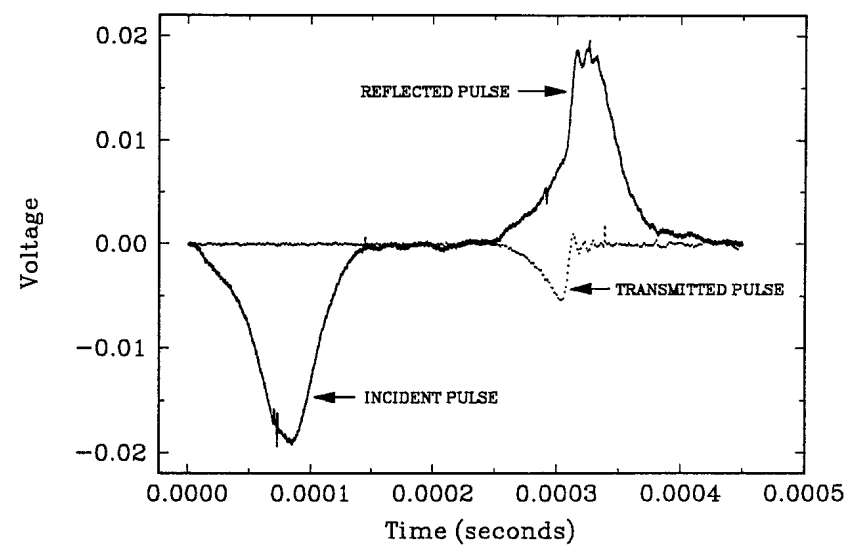

FIG. 3. Strain gage signals from dynamic compression test.

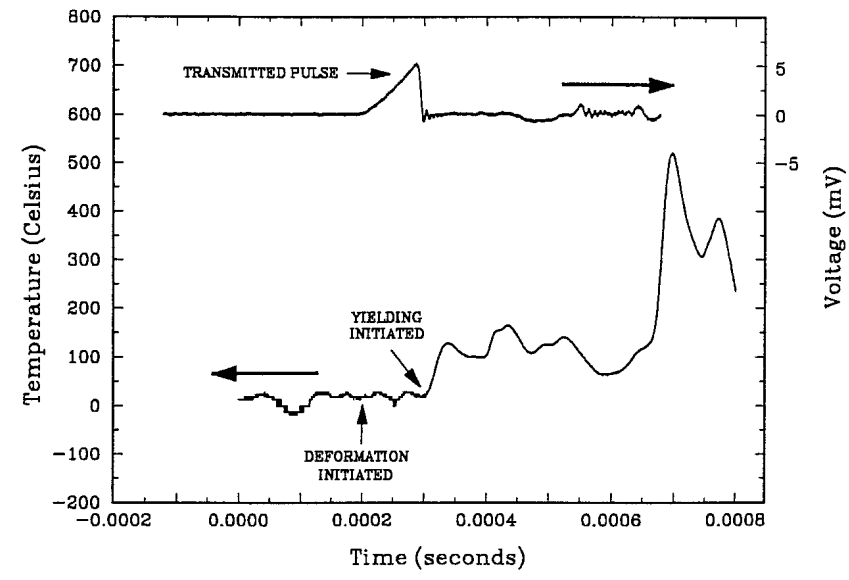

FIG. 4. Temperature and loading recorded from dynamic compression test.

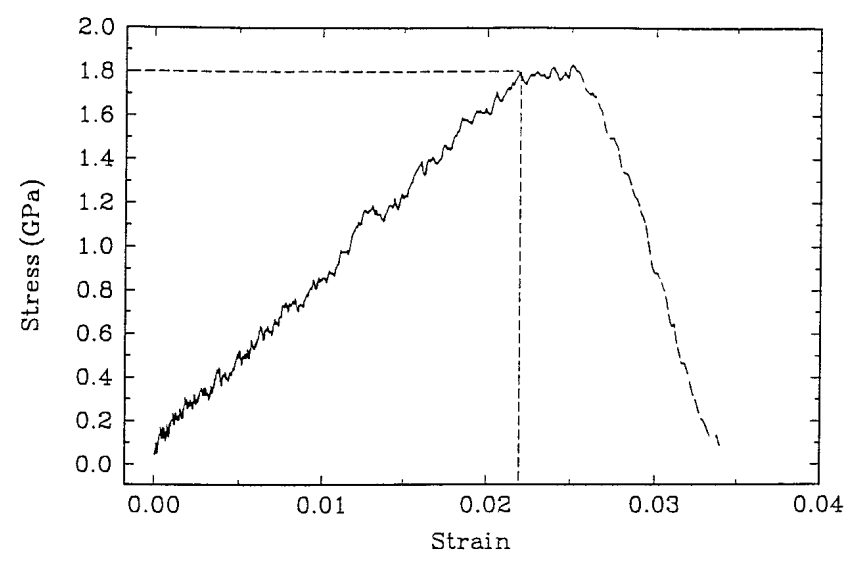

FIG. 5. Stress-strain curve reduced from strain gage signals in Fig. 5.

the shear band after deformation. This temperature is approaching the $\mathrm{Zr}_{41.25} \mathrm{Ti}_{13.75} \mathrm{Cu}_{12.5} \mathrm{Ni}_{10} \mathrm{Be}_{22.5}$ metallic glass melting point ${ }^{3}$ of $663{ }^{\circ} \mathrm{C}$, which would imply that the deformation within the shear band is so intense that the material within the band could be melting. It has also been observed that there is a temperature of $100{ }^{\circ} \mathrm{C}$ which is sustained for a period of time after the specimen has yielded. Since the homogeneous plastic deformation is too limited to account for this temperature rise, it could be due to the formation of micro-shear bands.

This is the first time transient thermal measurements have been made on a metallic glass during a shear banding event. However, more definite conclusions could be made about the origin of the thermal measurements if an array of detectors had been used to detect the inhomogeneous thermal distribution that would be associated with shear banding.

Evidence that localized melting may be responsible for the observed thermal measurements was obtained from a scanning electron micrograph of the failure surface, which can be seen in Fig 9. The only morphology that appears on the failure surface are vein-like 


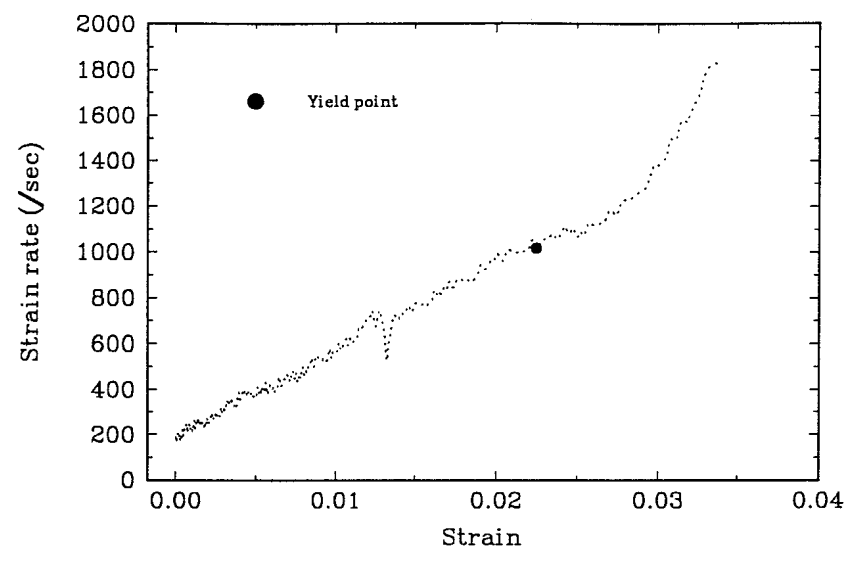

FIG. 6. Strain-strain rate curve reduced from strain gage signals in Fig. 5.

patterns. These features are identical to those observed on the failure surfaces of other metallic glass specimens tested in compression. An explanation for these features has been proposed which asserts that the viscosity of the material within a shear band is reduced considerably because of adiabatic heating generated by the enormous plastic deformations within the shear band which preceded catastrophic failure. ${ }^{9}$ As the material separates, pores are opened up, leaving behind the veinlike morphology. This hypothesis has been confirmed by shear experiments performed on a viscous material that has been placed between glass slides. ${ }^{10}$ After the slides are sheared apart, a vein-like morphology is revealed on the separated surfaces.

The dynamic compressive stress-strain response in Fig. 5 is very similar to the quasistatic compressive stress-strain response reported in Ref. 4. For the sake of comparison, the quasistatic compressive stress strain curve is shown in Fig. 10. The material exhibits an almost textbook case elastic-perfectly plastic response with yielding occurring at approximately $2 \%$ elastic

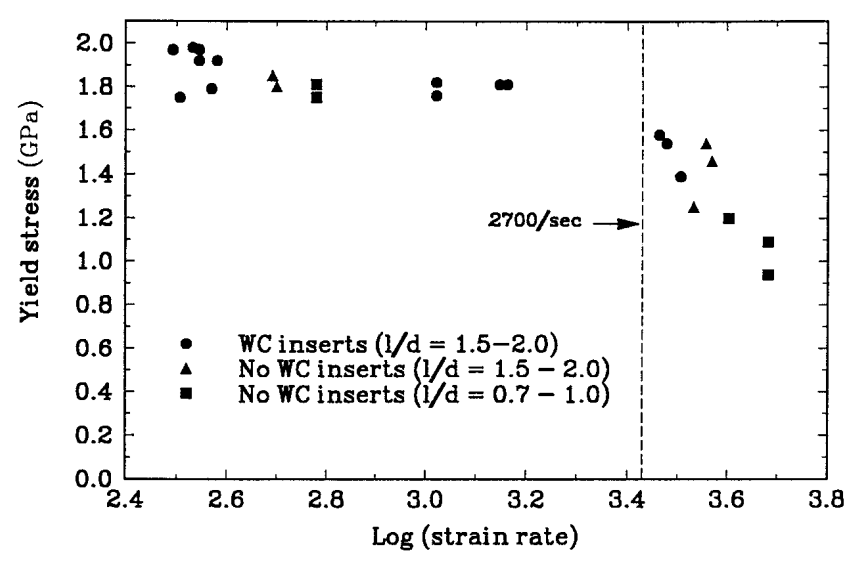

FIG. 7. Yield stress versus strain rate from the dynamic compression tests.

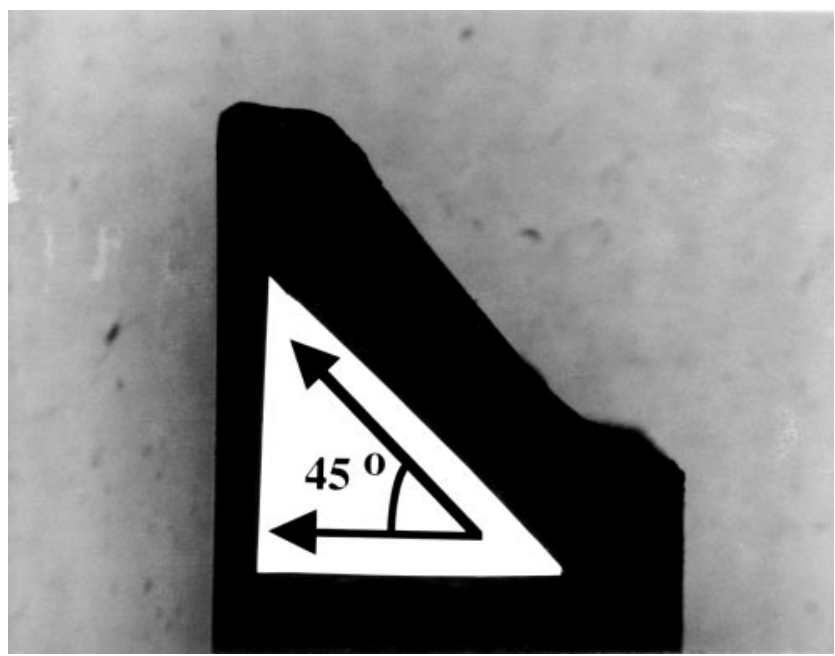

FIG. 8. Orientation of failure surface for $2.5 \mathrm{~mm}$ dynamic compression test specimen.

strain. From Fig. 7, it appears that the dynamic compressive yield stress is within $5 \%$ of the quasistatic value of $1.89 \mathrm{GPa}$ reported in Ref. 4 for strain rates below 1500/s. However, for strain rates greater than $3000 / \mathrm{s}$, the compressive yield stress appears to decrease monotonically with strain rate (strain rate softening). At first this was believed to have been due to adiabatic heating of the specimen; however, thermal measurements indicated that no heating occurred prior to the onset of yielding (Fig. 4).

During the dynamic compression tests, it was observed that the WC inserts were failing in tests conducted at strain rates above $3000 / \mathrm{s}$. If the WC inserts were failing before the specimens failed, then the observed strain rate softening could be due to the failure of the inserts. To test this theory, the WC inserts were removed and the compression tests repeated. By removing the inserts, the

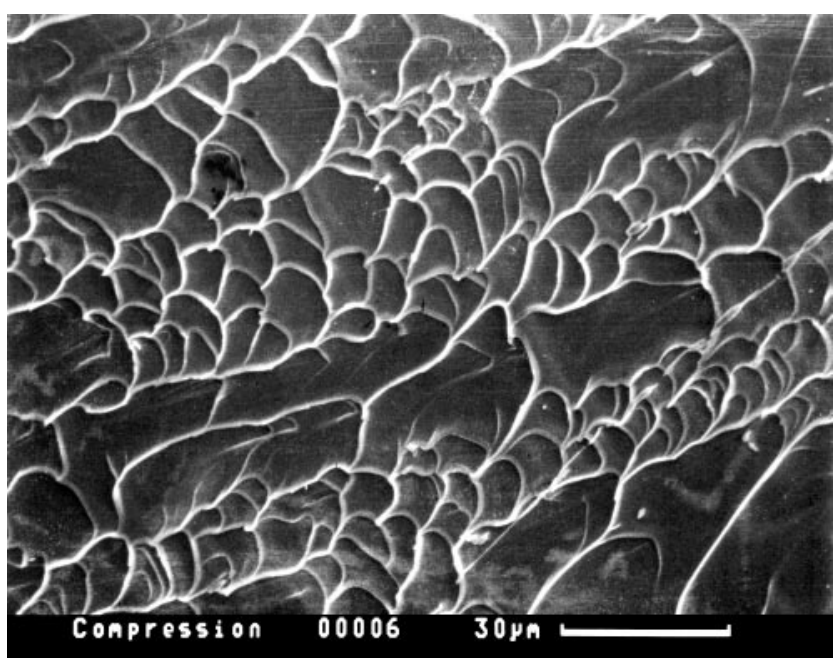

FIG. 9. Scanning electron micrograph of compressive failure surface. 


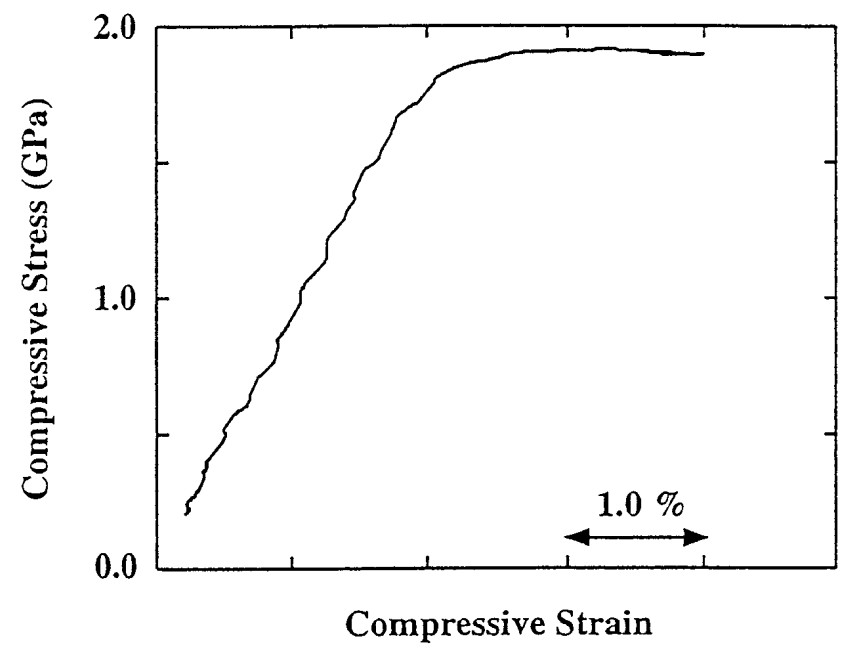

FIG. 10. Stress-strain curve from quasistate compression test.

stress concentrations at the end of the specimen might not be reduced which may cause permanent failure. The results from these tests in Fig. 7 indicate that removing the WC inserts did not alter the observed yield stress of the specimens at strain rates below $1500 / \mathrm{s}$, while still exhibiting the same softening behavior above 3000/s. Therefore, it was decided that the WC inserts should be removed from the dynamic compression tests since they were not affecting the test results.

No other observations were made during the dynamic compression tests that might explain the apparent strain rate softening. Therefore, it became necessary to reassess the conditions under which the dynamic compression tests were performed to rule out any possible artifacts in the testing system that might be responsible for the apparent strain rate softening.

There are three basic, underlying assumptions used in the reduction of split Hopkinson pressure bar test data. They are as follows: (i) The specimen undergoes homogeneous deformation during the experiment. (ii) The various stress pulses (e.g., reflected and transmitted) encountered in the split Hopkinson bar undergo minimal dispersion. (iii) The bars remain elastic at all times, and the ends of the bars in contact with the specimen remain flat and parallel throughout the experiment.

For the strain rates above $3000 / \mathrm{s}$, the magnitude of the incident stress pulse was $400 \mathrm{MPa}$, well below the $2.5 \mathrm{GPa}$ yield stress of the elastic bars. Therefore, assumption (iii) was not violated. Verifying that the other two assumptions were not violated required using analyses developed by Ravichandran and Ghatuparthi. ${ }^{11}$

\section{LIMITING STRAIN RATE FOR A METALLIC GLASS SPECIMEN IN A KOLSKY BAR}

Ravichandran used a simple analysis to establish the conditions under which a specimen must be tested to obtain homogeneous deformations consistent with assumption (i). ${ }^{11}$ This analysis assumed that there must be some minimum time which was required for the waves reflected back and forth inside a specimen to equilibrate. For a specimen which exhibits a linearly elastic material response up to some failure strain $\epsilon_{f}$, the limiting strain rate $\dot{\epsilon}_{l}$ was derived to be:

$$
\dot{\epsilon}_{l}=\frac{\epsilon_{f} c}{\alpha L},
$$

where $c$ is the specimen's longitudinal bar wave velocity, $L$ is the length of the specimen, and $\alpha$ is a nondimensional parameter whose value depends only on the shape of the incident pulse.

From an analysis of one-dimensional wave propagation in the elastic bars and specimen using the method of characteristics, ${ }^{11}$ it was determined that for typical incident pulses the gradient of stress along the length of the specimen was within 5\% of the mean stress in the specimen when $\alpha=4$. This meant that for the metallic glass specimens used for the dynamic compression test, which had lengths of $5 \mathrm{~mm}$ or less and a longitudinal bar wave velocity of $3925 \mathrm{~m} / \mathrm{s}$ determined from ultrasonic measurements, ${ }^{4}$ the limiting strain rates were 3925/s when the failure strain was at least $2 \%$. Although this value is larger than the $3000 / \mathrm{s}$ strain rate at which the softening began to occur, it was still close enough that it was decided to repeat the tests using specimens with aspect ratios of 0.7 to 1 , which would increase the limiting strain rate to above $8000 / \mathrm{s}$ and eliminate any questions about the homogeneity assumptions.

It has already been observed quasistatically that by lowering the aspect ratio the yield stress increases because a plain strain stress state is approached. Therefore, the conditions appeared encouraging for these tests. In Fig. 7, it can be seen that the yield stress results from these tests were identical to the test results for the longer specimens. Thus, it was concluded that the plane strain stress state was not being approached as the aspect ratios of the test specimens were reduced below 1 and that the inhomogeneity of the stress field was not responsible for the apparent softening behavior.

\section{EFFECTS OF DISPERSION IN A KOLSKY BAR AT HIGH STRAIN RATES}

Ceramics have exhibited the same apparent strain rate softening behavior as the $\mathrm{Zr}_{41.25} \mathrm{Ti}_{13.75} \mathrm{Cu}_{12.5} \mathrm{Ni}_{10^{-}}$ $\mathrm{Be}_{22.5}$ metallic glass at strain rates above $2700 / \mathrm{s}^{12}$ Dispersion effects were also analyzed by Ravichandran and Ghatuparthi to determine if they would account for the apparent softening behavior of ceramics. ${ }^{11}$ Because of dispersion effects, the amplitude and duration of a stress pulse changes as it propagates in a bar. The extent of geometrical dispersion depends on the dominant frequency components contained in the pulse that is being 
propagated, which is determined by the duration and shape of the pulse. ${ }^{13,14}$

From Ravichandran's analysis, the effects of dispersion are minimized when the dominant frequency component in the frequency spectrum of the input pulse in a cylindrical bar is such that $a / \Lambda<0.1$. Here, $a$ is the radius of the bar and $\Lambda$ is the wavelength associated with the dominant frequency component. This condition can be rewritten as:

$$
\left(\frac{c_{0}}{c_{p}}\right) \frac{\omega}{\omega_{0}}<0.1
$$

where $\omega_{0}$ is the fundamental frequency associated with the bar, $\omega$ is the fundamental frequency of the pulse, $c_{p}$ is the characteristic propagation velocity for the given frequency, and $c_{0}$ is the longitudinal bar wave velocity. The fundamental frequency associated with the bar is given by:

$$
\omega_{0}=2 \pi\left(\frac{c_{0}}{a}\right) .
$$

The value of $c_{p}$ for large wavelengths can be obtained from the Rayleigh approximation ${ }^{15}$ :

$$
\frac{c_{p}}{c_{0}}=1-\nu^{2}\left(\frac{a}{\Lambda}\right)^{2}
$$

where $\nu$ is Poisson's ratio.

For the Kolsky bars used in the dynamic compression experiments, $a=6.35 \mathrm{~mm}, \nu=0.3$, and $c_{0}=$ $4970 \mathrm{~m} / \mathrm{s}$. Thus, plugging these values into Eq. (15) yields $\omega_{0}=4.92 \times 10^{6} \mathrm{rad} / \mathrm{s}$. Using the value for $c_{p}$ at $a / \Lambda=0.1$ from Eq. (16), a limiting value of $4.88 \times$ $10^{5} \mathrm{rad} / \mathrm{s}$ for the fundamental frequency of the pulse $(\omega)$ is obtained. The corresponding period of the pulse, $T$, can be calculated as follows:

$$
\omega_{0}=\frac{2 \pi}{T} .
$$

Assuming that the time to failure coincides with the peak of the input pulse, and from symmetry, the time to failure $t_{f}$ will be $T / 2$. The minimum time to failure $t_{f}$ should be at least $6.44 \mu \mathrm{s}$ to minimize dispersion. Using the failure strain of $2 \%$ for the $\mathrm{Zr}_{41.25} \mathrm{Ti}_{13.75^{-}}$ $\mathrm{Cu}_{12.5} \mathrm{Ni}_{10} \mathrm{Be}_{22.5}$ metallic glass, the limiting strain rate would be $3100 / \mathrm{s}$. This is approximately the strain rate at which the apparent strain rate softening begins. Since the reflected pulse is also affected by dispersion, it is not unreasonable to believe that the strain rates, like the stresses, are also underestimated. So, the true strain rates are probably much higher than $3000 / \mathrm{s}$, which would mean that the strain rate softening occurs well beyond the calculated limiting strain rate.

There are two methods to correct the dispersion problem. The first is to directly correct for the dispersion in the signal. ${ }^{15}$ This requires that a sufficient number of data points be sampled so that the contribution of each significant frequency component can be extracted by means of a Fourier transform. At the sampling rates used in the dynamic compression experiments, approximately 80 data points could be obtained for strain rates above 3000/s. Unfortunately, this is not enough data to characterized properly the signal in terms of its significant frequency components. Therefore, dispersion in the signal cannot be corrected directly.

The second method involves placing a strain gage on the surface of the specimen to measure directly the specimen strain. ${ }^{12}$ In order to interpret the corresponding stresses, a linearly elastic material response must be assumed using a Young's modulus ${ }^{4}$ of $93 \mathrm{GPa}$ to compute the stresses. This assumption appears to be reasonable given that the $\mathrm{Zr}_{41.25} \mathrm{Ti}_{13.75} \mathrm{Cu}_{12.5} \mathrm{Ni}_{10} \mathrm{Be}_{22.5}$ metallic glass exhibits linearly elastic behavior up to yield and that the ductility of the specimen is very limited.

A strain gage from JP Technologies was placed on the surface of a dynamic compression test specimen, and tests were repeated for strain rates above 3000/s and at approximately 230/s. For strain rates of $230 / \mathrm{s}$, the specimen did not fail and the deformation was purely elastic, as evidenced by a stress-strain response identical to the linear portion of the curve in Fig. 5. The strains were recorded from the specimen strain gage and compared with the strain derived from the transmitted pulse, assuming a linearly elastic response (Fig. 11). From these low strain rate results, it appears that the strains from the specimen strain gage are within $5 \%$ of the strains derived from the transmitted pulse with the greatest deviation occurring at the peak strain level. This is not unexpected since strain gage signals are rarely accurate to within $10 \%$ of the actual strain depending on the strength of the adhesion between the gage and the specimen surface. The strain rate was computed by curve fitting the linear portion of the strain signal from the specimen strain gage.

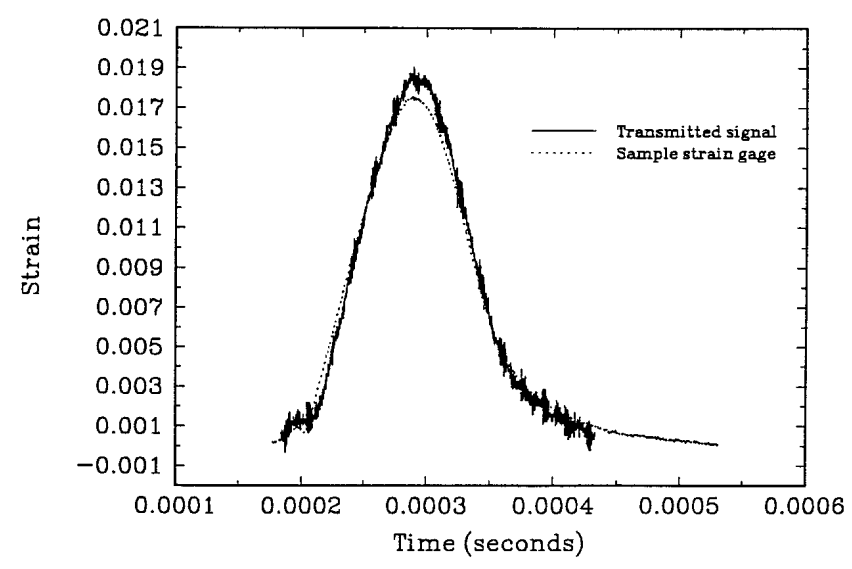

FIG. 11. Dynamic strain response from the specimen strain gage and derived from the transmitted signal for a strain rate of $230 / \mathrm{s}$. 
After elastically loading the specimen at low strain rates, the specimen was reloaded at a strain rate above 3000/s. A typical result can be seen in Fig. 12 where the strain rate was determined to be approximately 3400/s. From these results it is obvious that the strain derived from the transmitted signal does not correlate with the strain from the specimen strain gage. In fact, the strain from the strain gage has a bandwidth of approximately $10 \mu \mathrm{s}$, while the strain derived from the transmitted signal has a bandwidth of about $14 \mu \mathrm{s}$. This difference in bandwidths was also observed in high strain rate compression tests conducted on ceramics and attributed to dispersion effects.

The strain response from the specimen strain gage shows a dramatic increase in strain rate at approximately $2.0 \%$ strain, which is most likely due to the initiation of shear banding. Therefore, this was taken to be the yield strain of the specimen. The peak strain derived from the transmitted bar is approximately $1.3 \%$, a value $35 \%$ below the yield strain. The differences in peak strain were also observed in the high strain rate experiments conducted on ceramics and attributed to dispersion effects.

The yield stress was therefore calculated to be approximately $1.9 \mathrm{GPa}$, which is within $5 \%$ of the value derived from the transmitted signal at strain rates below 3000/s. This yield stress can be seen in Fig. 13 plotted against the data from Fig. 7 along with another data point obtained using a strain gage on a specimen tested at a strain rate of approximately 4400/s that had not been prestrained. The data points obtained using specimen strain gauges are larger than any of the yield stresses derived from the transmitted signal at strain rates above $3000 / \mathrm{s}$, clearly indicating that dispersion effects are responsible for the apparent softening behavior observed at strain rates above 3000/s.

Although peak strains of $3.5 \%$ were measured from the specimen strain gage, it is difficult to interpret the corresponding load levels. Obviously, if shear banding

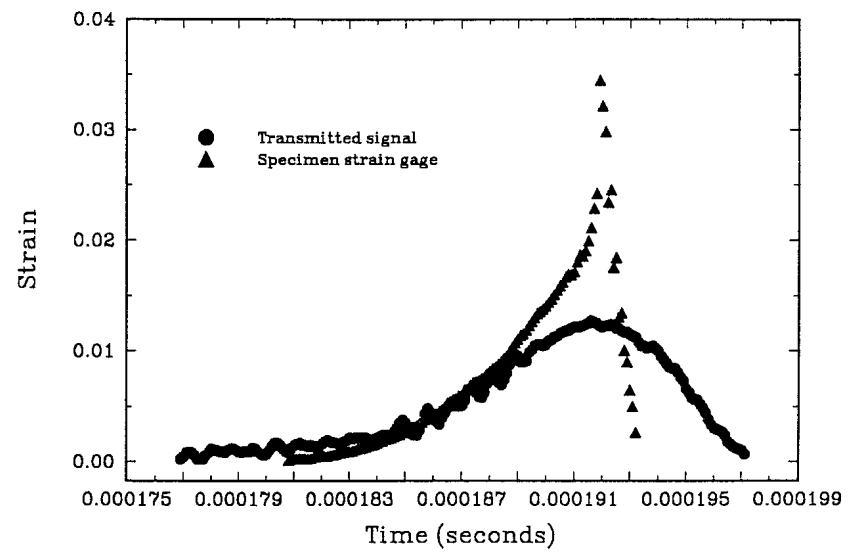

FIG. 12. Dynamic strain response from the specimen strain gage and derived from the transmitted signal for a strain rate of 3400/s.

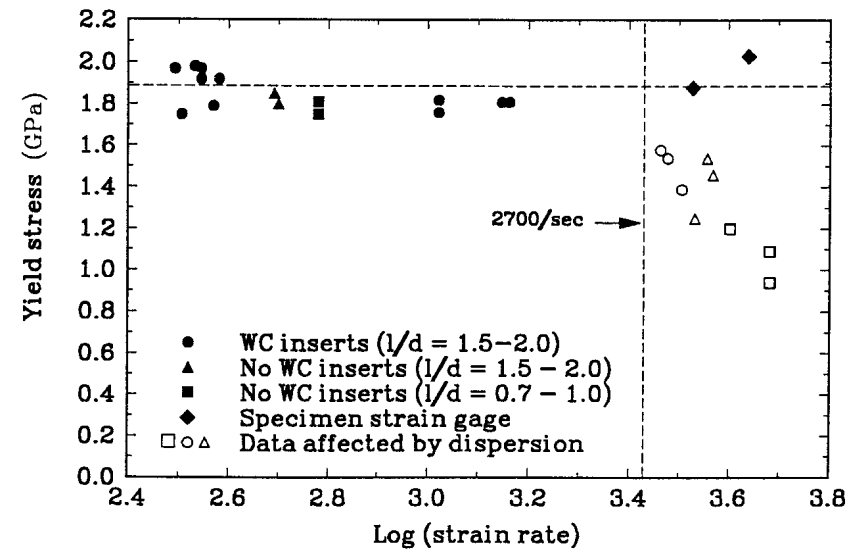

FIG. 13. Data from Fig. 9 plotted against specimen strain gage data.

has occurred, the stresses cannot be derived assuming a linearly elastic behavior. Furthermore, the strain would appear to be accumulated at local rates within the shear bands of approximately $30,000 / \mathrm{s}$. It has been postulated that strain rates of this order or higher might occur inside a shear band during dynamic deformation. Although it is unreasonable to assume that the strain gage could resolve strains inside a shear band, it does indicate that there is some type of post-yield behavior such as shear band deformation occurring.

While the strain derived from the transmitted signal is affected by dispersion, the strain derived from the reflected signal does not appear to be as sensitive (Fig. 14). In fact, up to yielding the two signals appear to be identical. This would indicate that although the yield stresses in Figs. 7 and 13 might be affected by dispersion at strain rates above $3000 / \mathrm{s}$, the strain rates should be reasonably accurate. Given that the typical reflected signal is similar to the incident signal, as seen in Fig. 3, with a period twice as large as that of the transmitted signal, it appears reasonable that the reflected signal would be insensitive to dispersion.

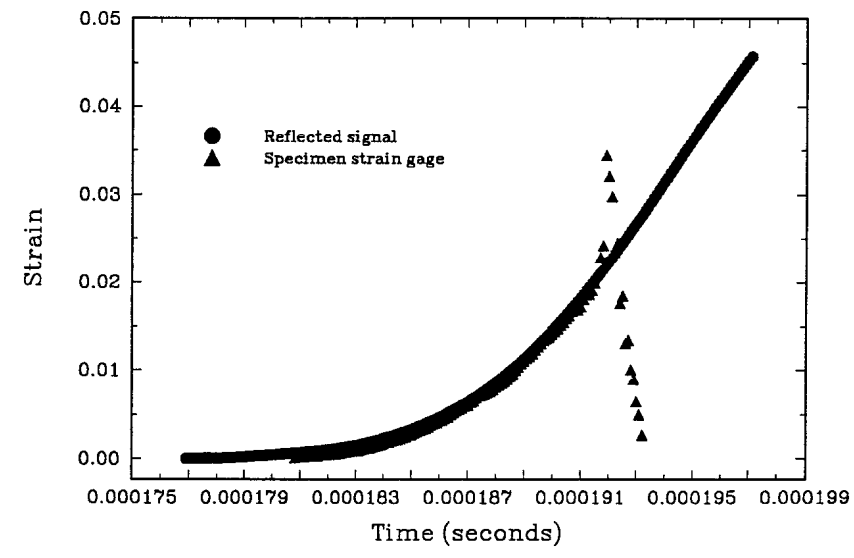

FIG. 14. Dynamic strain response from the specimen strain gage and derived from the reflected signal for a strain rate of 3200/s. 


\section{CONCLUSIONS}

For the first time, dynamic tests have been conducted on a metallic glass at strain rates on the order of $10^{2}-10^{3} / \mathrm{s}$. From these tests, it appears that the metallic glasses retain their elastic-perfectly plastic material behavior at high strain rates. Furthermore, the yield stress of the $\mathrm{Zr}_{41.25} \mathrm{Ti}_{13.75} \mathrm{Cu}_{12.5} \mathrm{Ni}_{10} \mathrm{Be}_{22.5}$ metallic glass was not found to depend on strain rate. This result is not unexpected since the micromechanisms for yielding in metallic glasses are much different than in conventional polycrystalline metals and ceramics, where the growth and motion of defects like dislocations and microcracks are sensitive to strain rate.

Thermal measurements of shear banding processes in metallic glasses have been made for the first time. These measurements indicate that temperature increases due to adiabatic heating occur only after the onset of inhomogeneous deformation. Furthermore, temperatures near the melting point of the $\mathrm{Zr}_{41.25} \mathrm{Ti}_{13.75} \mathrm{Cu}_{12.5} \mathrm{Ni}_{10} \mathrm{Be}_{22.5}$ metallic glass may be approached within shear bands after the specimen has failed. This is consistent with SEM observations of a vein-like morphology on the failure surface which is attributed to localized melting during shear banding failure. Since only a single detector was used for the thermal measurements, it was impossible to identify the detailed nature of the thermal distribution near the shear band.

\section{REFERENCES}

1. G. I. Taylor, Proc. R. Soc. London A194, 289 (1948).

2. H. Kolsky, Proc. R. Soc. London B62, 676 (1949).

3. A. Peker and W. L. Johnson, Appl. Phys. Lett. 63, 2342 (1993).

4. H. A. Bruck, T. Christman, A.J. Rosakis, and W.L. Johnson, Scripta Metall. Mater. 30, 429 (1994).

5. C. F. Cline and J.E. Reaugh, J. de Phys. IV 1, 139 (1991).

6. A. T. Zehnder and A.J. Rosakis, J. Mech. Phys. Solids 39, 385 (1991).

7. A. J. Rosakis, J. J. Mason, and G. Ravichandran, unpublished.

8. P. S. Follansbee, Metals Handbook (American Society of Metals, Metals Park, OH, 1985), Vol. 8, p. 198.

9. H. J. Leamy. H. S. Chen, and T. T. Wang, Metall. Trans. 3, 699 (1972).

10. C. A. Pampillo and A.C. Reimschuessel, J. Mater. Sci. 9, 718 (1974).

11. G. Ravichandran and S. Ghatuparthi, J. Am. Ceram. Soc. 77, 263 (1994).

12. J. M. Staehler, W.W. Predebon, B. J. Pletka, and J. Lankford, J. Am. Ceram. Soc. 76, 536 (1993).

13. R. A. Davies, Philos. Trans. R. Soc. London A240, 375 (1948).

14. P. S. Follansbee and C. Frantz, J. Eng. Mater. Technol. 105, 61 (1983).

15. H. Kolsky, Stress Waves in Solids (Dover Publications, New York, 1963). 